\title{
Tunable and Growing Network Generation Model with Community Structures
}

\author{
Muhammad Qasim Pasta \\ Karachi Institute of Economics and Technology \\ Karachi, Pakistan \\ Email:mqpasta@pafkiet.edu.pk
}

\author{
Zohaib Jan \\ Shaheed Zulfikar Ali Bhutto Institute of Science and \\ Technology \\ Karachi, Pakistan \\ Email:zohaib.jan@ szabist.edu.pk
}

\author{
Arnaud Sallaberry \\ LIRMM, Université Montpellier 3, \\ Montpellier, France \\ Email: arnaud.sallaberry@lirmm.fr
}

\author{
Faraz Zaidi \\ Karachi Institute of Economics and Technology \\ Karachi, Pakistan \\ Email: faraz@pafkiet.edu.pk
}

\begin{abstract}
Recent years have seen a growing interest in the modeling and simulation of social networks to understand several social phenomena. Two important classes of networks, small world and scale free networks have gained a lot of research interest. Another important characteristic of social networks is the presence of community structures. Many social processes such as information diffusion and disease epidemics depend on the presence of community structures making it an important property for network generation models to be incorporated.
\end{abstract}

In this paper, we present a tunable and growing network generation model with small world and scale free properties as well as the presence of community structures. The major contribution of this model is that the communities thus created satisfy three important structural properties: connectivity within each community follows power-law, communities have high clustering coefficient and hierarchical community structures are present in the networks generated using the proposed model. Furthermore, the model is highly robust and capable of producing networks with a number of different topological characteristics varying clustering coefficient and inter-cluster edges. Our simulation results show that the model produces small world and scale free networks along with the presence of communities depicting real world societies and social networks.

\section{INTRODUCTION}

Traditionally graph and network studies were made using regular and random graphs [1] until the late 1990's when two ground breaking discoveries were made about real world networks. The presence of low average path lengths and high clustering coefficients lead to the discovery of small world networks [2] and the study of degree distribution following power-law lead to the discovery of scale free networks [3]. These topological characteristics are commonly present in many real world networks such as social networks [4], biological networks [5] and information networks [6].

The discovery of small world and scale free properties have catalysed the research in the area of developing new graph and network generation models as networks with these properties appear readily across different and contrasting domains. This research area presents interesting challenges and new horizons for researchers to develop theories, models and algorithms based on the simulation and modeling of networks with domain dependent as well as domain independent characteristics. Furthermore, these models help us understand the underlying processes and structural changes taking place in many diverse real world networks.

An important characteristic of these networks is the presence of community structures. Networks in general and social networks in particular highly depend on a society based structure where groups of people are very well connected to each other and sparsely connected to people from other groups [7]. This phenomena has been observed in networks from many different domains such as computer networks [8], biological networks [9] and maritime transportation networks $[10]$. A number of network studies depend on the underlying community structure present in a network [11]. For example, [12] studied communities of scientists and the role of information diffusion in the creation of knowledge. [13] studies the effects of communities on the immunity coverage required to prevent disease epidemics in societies. Tunable methods that can generate networks with desired network characteristics and the presence of communities can be very useful in such studies as they can provide a benchmark for empirical evaluation. Researchers [14], [15] have proposed different models to generate networks with community structures in an attempt to generate networks which are topologically similar to real world networks.

An important feature often overlooked by different network generation models with community structures is the topological structure of a community itself. Usually models propose increased intra-community links and reduced intercommunity links but the connectivity within a community is ill defined or follow only power-law degree distribution. The major contribution of the model is that we maintain three important topological characteristics within each community:

- The degree distribution of nodes follows a power-law.

- The clustering coefficient is high.

- Each community can be further divided into subcommunities, i.e. there are hierarchical communities 
in the network

Each of the above characteristics has associated social semantics. Consider a co-authorship network where researchers collaborate to author manuscripts. Each research group represents a community as members regularly collaborate to increase intra-cluster edges which in turn results in high clustering coefficient. Subsequently, this research group also belongs to the community of researchers working in the same area across different research labs and different countries. These researchers collaborate less frequently but have still more edges when compared with research groups working in different domains. This creates a hierarchical community structure in the co-author network as argued by other researchers as well [16]. Each research group is usually headed by a senior professor with research publications, which means that a senior professor will have a high number of co-authors. Structurally this implies that the senior professor will have many edges connecting it to many authors. Usually each research group has a few senior researchers with high publication profile and the rest of the team comprises of researchers with low publications. These researchers are often associated with the senior professors while authoring an article justifying that every community demonstrates scale invariance power-law. Similarly the group dynamics and synergy is reflected by the people within a research group collaborating to author manuscripts. This results in a high number of triad formation which in turn results in high clustering coefficient for members of a research groups depicting a community.

The contribution of this paper is that we propose a new network generation model. The proposed model is inspired by [17] to generate networks with small world and scale free properties where we modify it to introduce community structures. The model caters the three described features present in community structures which is fundamental to many real world networks and specially in the case of social networks. Parameters to control inter-cluster connectivity and triad formation gives us more flexibility over the generation process and thus enables us to generate networks with desired properties. The networks produced using the proposed algorithm also exhibit small world and scale free properties. The model is tunable and robust as it can be used to generate a variety of networks by varying different parameters such as only scale free networks with community structures and networks with varying intercluster edges.

The rest of the paper is structured as follows: In the next section, we review the literature related to network generation models. Section III describes the proposed model whereas section IV analyses and explains the use of different parameters to generate networks with varying structural properties. Section $V$ presents the results of the evaluation of the networks generated using the proposed model satisfying the small world and scale free properties with clear community structures. Finally we conclude in section VI giving future research directions.

\section{RELATED WORK}

We divide the literature review into two logical subsections.

\section{A. Models for Random, Small World and Scale Free Networks}

Earlier studies related to network models were focussed on generating random graph. Most notable work of all is the graph generation model by [1]. Molloy and Reed [18] proposed a model to generate graphs with desired degree distribution. Watts and Strogatz proposed the famous model to generate small world networks [2] where the algorithm starts with a regular graph and random rewiring of edges based on some probability results in a small world graph with small average path length and high clustering coefficient. Albert and Barabasi introduced another important model [3] based on preferential attachment to generate scale free networks.

Since the discovery of small world and scale free networks, a number of network models have been proposed to generate networks with these two properties. Most of these models are variants of the two basic models [2], [3] discussed above. For example Holme and Kim [17] introduce a triad formation step after the preferential attachment step in [3] which creates triads in the network increasing the overall clustering coefficient. Other variants such as [5], [19]-[24] produce networks by introducing triads one way or the other and nodes connect using the preferential attachment rule to have a scale free degree distribution.

Another approach for generating small world and scale free networks is the use of n-partite structure. Newman et al. [25] study a network generation model with arbitrary degree distribution. The goal is to generate affiliation networks similar (such as co-authorship network [6]) using random bipartite graphs. Guillaume and Latapy [26] also used a similar idea as they identify bipartite graphs as an underlying structure for networks with small world and scale free properties. Bu et al. [27] used a n-partite structure, which is simply a generalization of the earlier proposed models. Good references on network generation models can be found in [28]-[30].

\section{B. Models for Networks with Community Structures}

Li and Chen [31] introduced a model for weighted evolving networks with community structures. The model incorporated three types of power-law distributions, first on the node degree, second on link weights and third on node strengths along with the presence of clear communities. The model does not produce networks with high clustering coefficient as nodes within a community do not follow triadic closure property.

Xie et al. [32] proposed a community-based evolving network model where they focus on the cumulative distribution of community sizes which also follows power-law in real world networks. As a result, when new connections between communities are added, or a new node to an existing community is added, communities with larger sizes are selected preferentially.

Zhou et al. [33] identify two important topological characteristics, first, intra-cluster connections are very dense as compared to inter-cluster connections and second, size of communities often follows a power-law just as [32] proposed. Based on these characteristics, they propose a weighted growing model with power-law distributions of community sizes, node strengths, and link weights. 
Kumpula et al. [34] utilize the concepts of cyclic closure and focal closure from sociology to propose a model to generate a weighted network with communities. New links are created preferably through strong ties which make these links more stronger. The model also allows the removal of nodes to mimic real world scenarios where nodes may leave a network.

$\mathrm{Xu}$ et al. [35] introduce a model with communities that gives a realistic description of local events using three processes, adding new intra-community nodes, new intracommunity links or new inter-community links. The model uses preferential attachment mechanism resulting in power law degree distribution but since the intra-community links only connect on the basis of node degree, the network lacks triads, producing networks with low clustering coefficients.

Lancichinetti and Fortunato [15] propose an algorithm to generate benchmarks to test clustering algorithms for directed/undirected and weighted/unweighted graphs with optional overlapping communities. This algoritm produces networks following power-law distribution for node degree as well as community sizes. They do not address the internal structure of each community as we do in this paper.

Badham and Stocker [29] propose a spatially constructed algorithm to generate networks with tunable degree distribution, clustering coefficient and assortativity with the objective that such models should be flexible to generate networks with varying values of these properties giving more control over the generation process. They do not explicitly include the generation of community structures in their model.

Ren et al. [36] study the connecting patterns among existing papers in co-authorship networks and highlight that existing models cannot correctly model high clustering in such networks. Their proposed model can generate networks with power-law degree distribution, high clustering coefficient and the size distribution of co-citation clusters as observed in coauthorship networks.

Moriano and Finke [37] also propose a model with small world and scale free properties along with groups of nodes densely connected to each other and sparsely connected with other nodes. The model helps to explain networks with extended power law degree distributions and clustering coefficient that does not diminish as the size of the network grows very large. The connectivity of new nodes probabilistically chooses nodes of same type or different type to form community structures.

Zaidi [30] proposed a model to generate clustered small world networks. The author first demonstrates that small world networks can be produced from completely random graphs by introducing a little order in them, which is a contrasting approach to the famous model of [2]. The further extends this model to generate clustered networks with small world properties where communities connect randomly to other communities. The model does not generate scale free networks.

Zaidi et al. [38] also proposed a static network generation model with community structures i.e nodes added at the start remain the same throughout the algorithm and only edges are rewired to create communities. The model is probabilistic and increases the edge connectivity among nodes closer to each other and reduces edges among nodes far apart in the network.
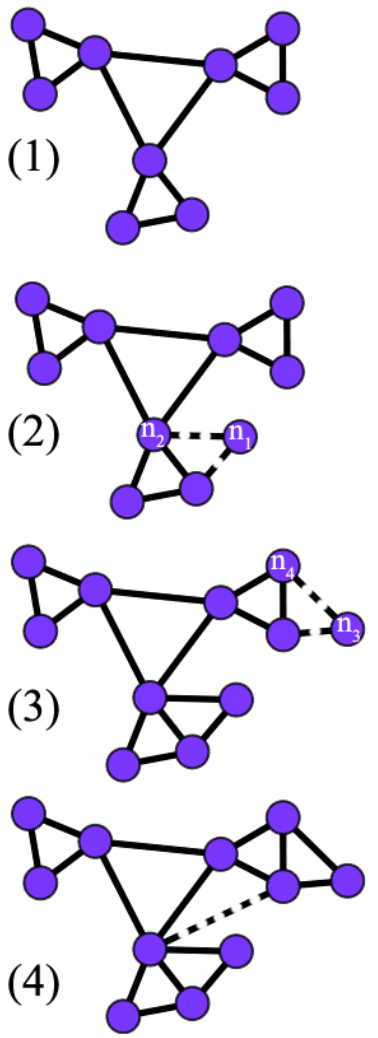

Fig. 1. Steps of the proposed model. (1) Step 1 with $c=3$ triads where every triad is connected to every other triad. (2) Step 2 A new node $n_{1}$ is added and forms a triad with probability $P_{t}$ with a neighbor of $n_{2}$. (3) Step 4 A new node $n_{3}$ is added and forms a triad with probability $P_{t}$ with a neighbor of $n_{4}$. (4) Step 6. The communities of newly added nodes $n_{1}$ and $n_{3}$ which are selected on the basis of preferential attachment forms an inter-cluster edge with probability $P_{c}$.

But the process is not a growing one, and it is not parametrized to generate desirable clustered network as compared to the model proposed in this study. Furthermore this model does not ensure the presence of the three structural properties for a community discussed in the introductory section of this paper.

All the different models for generating networks with community structures discussed above do not focus on the internal structure of communities just as we do in this paper. We focus on three structural properties present in our society and propose a model to simulate these properties.

\section{PROPOSED MODEL}

The model takes as input the desired number of nodes in the network $n$, the number of edges for each newly added node $m$, the minimum number of communities $c$, the probability of triad formation $P_{t}$ and probability $P_{c}$ of having inter-cluster edges. The model starts with an empty network. Rest of the steps are explained below:

1) Add $c$ triads representing $c$ communities in the network. Each node in a triad belongs to the community of the triad. Every community thus created is then connected to every other community. An edge is created between randomly selected nodes from different 
communities. This step generates a graph as shown in figure 1 (1).

2) Add a new node $n_{1}$ and connect it to an existing node $n_{2}$ which is selected on the basis of preferential attachment. Now $n_{1}$ belongs to the community of $n_{2}$ as shown in figure 112).

3) With probability $P_{t}$, either $n_{1}$ connects to $m-1$ preferentially selected neighbors of $n_{2}$ belonging to the same community as $n_{2}$ forming a triad or $n_{1}$ connects to other nodes preferentially selected from the community of $n_{2}$ (which might not necessarily be a neighbor of $n_{2}$ ). Figure 1(2) shows that the newly added node connected to one of the neighbors of $n_{2}$.

4) Add a new node $n_{3}$ and connect it to an existing node $n_{4}$ which is again selected on the basis of preferential attachment. Now $n_{3}$ belongs to the community of $n_{4}$. We make sure that $n_{3}$ does not belong to the community of the node added in the previous nodeaddition step to the network. Figure 113) shows this step.

5) With probability $P_{t}$, either $n_{3}$ connects to $m-1$ preferentially selected neighbors of $n_{4}$ belonging to the same community as $n_{4}$ forming a triad or $n_{3}$ connects to other nodes preferentially selected from the community of $n_{4}$ ( which might not necessarily be a neighbor of $n_{4}$ ), as shown in Figure 11(3).

6) With probability $P_{c}$, add an edge between two preferentially selected nodes belonging to the two communities to which nodes were added in the previous steps, which is the communities of $n_{2}$ and $n_{4}$ as shown in figure 1.4).

7) Repeat from step 4 until number of nodes in the network becomes $n$.

\section{Discussion}

As described above, the model uses five parameters, $n, m, c, P_{t}, P_{c}$. The parameter $n$ defines the number of nodes desired in the final network and $m$ defines the number of connections each newly added node will have in the network (except for the nodes added in the initial triads in step 1).

Preferential Attachment for individual nodes: The probability of a new node preferentially selecting an existing node $n_{i}$ from the set of current vertices $V$ is a function of the degree of node $n_{i}$ which can be calculated using:

$$
P\left(n_{k}\right)=\frac{\operatorname{degree}\left(n_{k}\right)}{\sum \operatorname{degree}\left(n_{j}\right)}, \forall j \in V
$$

This ensures that the nodes are selected based on preferential attachment.

Preferential Attachment for each community: The probability that a new node selects a community $c_{k}$ to attach can be estimated as:

$$
P\left(c_{k}\right)=\frac{\sum \operatorname{degree}\left(n_{i}\right)}{\sum \operatorname{degree}\left(n_{j}\right)}, \forall i \in c_{k}, \forall j \in V
$$

As the network grows, the community sizes vary as a

\begin{tabular}{|c|c|c|c|c|}
\hline $\begin{array}{l}\text { S.No } \\
\text { Key }\end{array}$ & $\begin{array}{c}\text { Number } \\
\text { of Nodes }\end{array}$ & $\begin{array}{c}\text { Initial Number } \\
\text { of Triads }\end{array}$ & $\begin{array}{c}\text { Probability of } \\
\text { Triad Formation }\end{array}$ & $\begin{array}{c}\text { Probability of } \\
\text { Inter-Cluster Edges }\end{array}$ \\
\hline 1 & 1000 & 10 & 0.5 & 0.01 \\
\hline 2 & 1000 & 10 & 0.5 & 0.10 \\
\hline 3 & 1000 & 10 & 0.5 & 0.5 \\
\hline 4 & 1000 & 10 & 0.5 & 1.0 \\
\hline 5 & 1000 & 10 & 1.0 & 0.01 \\
\hline 6 & 1000 & 10 & 1.0 & 0.10 \\
\hline 7 & 1000 & 10 & 1.0 & 0.5 \\
\hline 8 & 1000 & 10 & 1.0 & 1.0 \\
\hline 9 & 1000 & 20 & 0.5 & 0.01 \\
\hline 10 & 1000 & 20 & 0.5 & 0.10 \\
\hline 11 & 1000 & 20 & 0.5 & 0.5 \\
\hline 12 & 1000 & 20 & 0.5 & 1.0 \\
\hline 13 & 1000 & 20 & 1.0 & 0.01 \\
\hline 14 & 1000 & 20 & 1.0 & 0.10 \\
\hline 15 & 1000 & 20 & 1.0 & 0.5 \\
\hline 16 & 1000 & 20 & 1.0 & 1.0 \\
\hline 17 & 10000 & 10 & 0.5 & 0.01 \\
\hline 18 & 10000 & 10 & 0.5 & 0.10 \\
\hline 19 & 10000 & 10 & 0.5 & 0.5 \\
\hline 20 & 10000 & 10 & 0.5 & 1.0 \\
\hline 21 & 10000 & 10 & 1.0 & 0.01 \\
\hline 22 & 10000 & 10 & 1.0 & 0.10 \\
\hline 23 & 10000 & 10 & 1.0 & 0.5 \\
\hline 24 & 10000 & 10 & 1.0 & 1.0 \\
\hline 25 & 10000 & 20 & 0.5 & 0.01 \\
\hline 26 & 10000 & 20 & 0.5 & 0.10 \\
\hline 27 & 10000 & 20 & 0.5 & 0.5 \\
\hline 28 & 10000 & 20 & 0.5 & 1.0 \\
\hline 29 & 10000 & 20 & 1.0 & 0.01 \\
\hline 30 & 10000 & 20 & 1.0 & 0.10 \\
\hline 31 & 10000 & 20 & 1.0 & 0.5 \\
\hline 32 & 10000 & 20 & 1.0 & 1.0 \\
\hline
\end{tabular}
function of the high degree nodes present in that community.
TABLE 1. THE TABLE SHOWS THE 24 DIFFERENT COMBINATIONS POSSIBLE FOR 4 PARAMETERS AND DIFFERENT POSSIBLE VALUES. THE SERIAL NUMBER WILL BE USED AS A KEY TO IDENTIFY NETWORKS AND THEIR PARAMETERS USED.

This ensures that communities of different sizes evolve in the network where the degree distribution of community sizes follow scale free behavior. This is because new nodes select a node to attach based on its degree, which in turn implies that a community is selected based on preferential attachment of nodes present in a community as shown in the above equation.

Minimum Number of Communities $c$ : This parameter controls the minimum number of communities we want to generate in the network. Further Communities and subcommunities form as order emerges from the connectivity of new nodes entering the network probabilistically. As the number of nodes increases, sub-communities increase depicting the natural evolution process of communities in real world networks. A simple variation for this parameter would be to use the value 1 signifying only 1 community, along with the triad formation step using $P_{t}$, the behavoir of the network would be the same as the model proposed by [17]. Small sub-communities will still form in this network but they will not be clearly separable. Another important variation would be if we use $c=1, m=1$ and $P_{t}=0$ i.e. eliminating the triad formation step, the model generates random scale free networks similar to [3].

Probability of Triad Formation $P_{t}$ : This parameter controls the presence of triads in the network. The triad formation step is performed with a probability $P_{t}$, or a preferential attachment step is performed with probability $1-P_{t}$ instead of triad formation step. Both the triad formation step or preferential attachment step, the new node is only connected to nodes from the same community. A value of $P_{t}=0$ means 

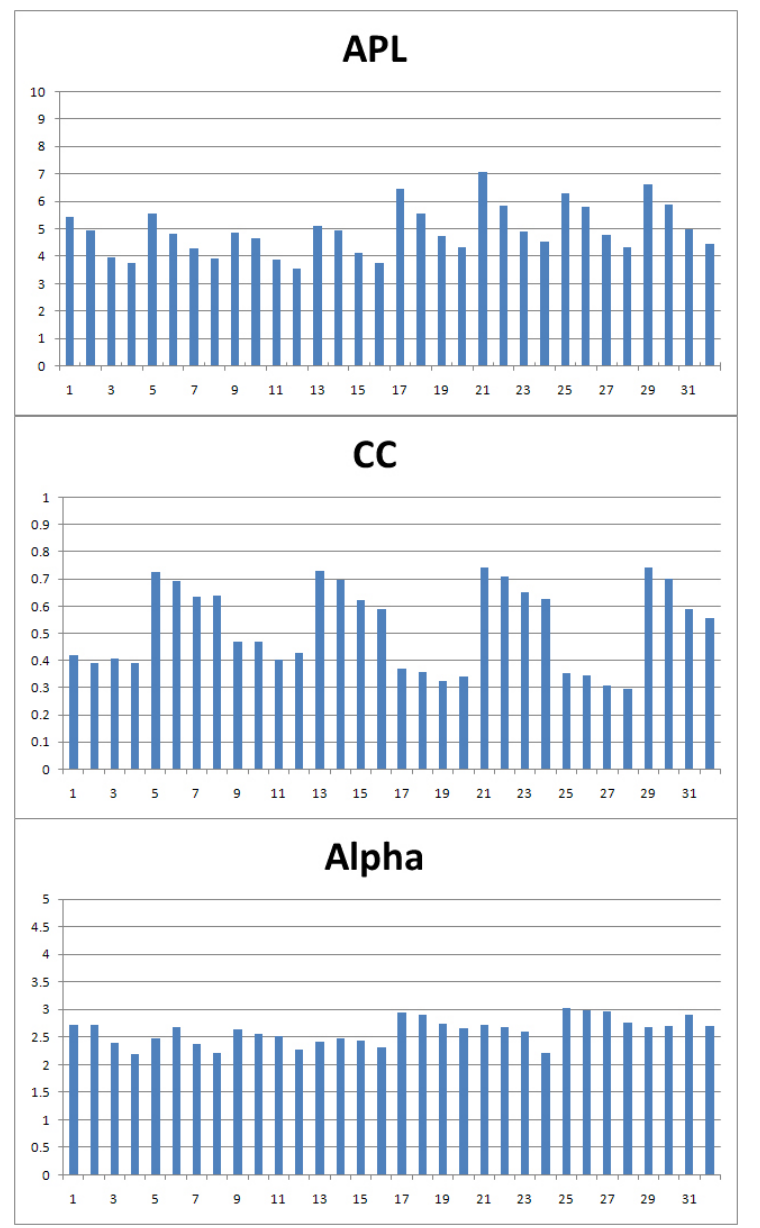

Fig. 2. Different metrics calculated for the 32 generated graphs showing that the graphs are indeed small world and scale free. (a)APL=Average Path Length (b) $\mathrm{CC}=$ Clustering Coefficient (c)) Alpha=Power-law coefficient.

that in steps 3 and 5, triads are not formed, as a result of which the overall clustering coefficient remains quite low. The network thus generated is a random scale free network with communities. A value of $P_{t}=1$ would mean that every node added to this network with $m$ edges, forms triads for every edge making the overall clustering coefficient quite high as is the case for small world networks.

Probability of Inter-cluster Edges $P_{c}$ : The inter-cluster density is controlled through this probability. A value of $P_{c}=$ 0 means that no further intra-cluster edges would be added to the network as described in step 6. This results in well separated communities with exactly two intra-cluster edges for each community which were added in step 1 . These edges are added so that the final network obtained, remains a connected network. A value of $P_{c}=1$ results in high inter-cluster edges making it difficult to distinguish communities structurally.

We demonstrate the effects of varying these parameters empirically in the next section as we generate numerous networks using the proposed model.

\section{EXPERIMENTATION AND RESULTS}

We tested the proposed model with the parameter values of $n=\{1000,10000\}, c=\{10,20\}, P_{t}=\{0.5,1.0\}$ and

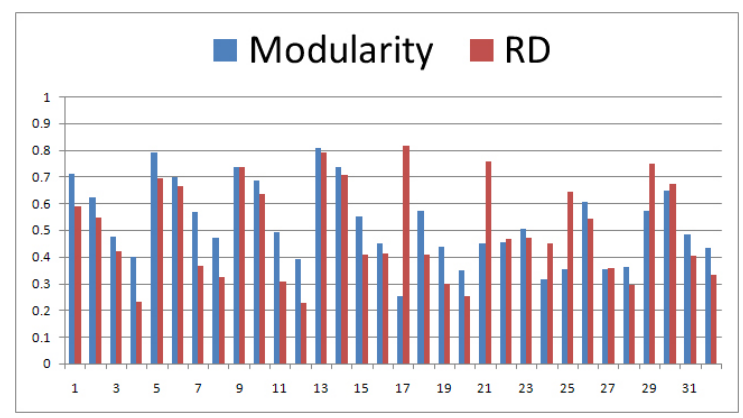

Fig. 3. Values of Modularity (Q)-Blue Bar and Relative Density (RD)-Maroon Bar, obtained after running a clustering algorithm on the generated networks. High values suggest the presence of community structures in the generated network.

$P_{c}=\{0.01,0.1,0.5,1.0\}$ which makes 32 different combinations. We used $m=\{2\}$ for all performed experiments which means that every new node entering the network has degree 2 to start with, which increases probablistically as other new nodes select previously added nodes as their connections. Table [1] shows all these combinations and a key is assigned to each combination to uniquely identify a network and the parameters used to generate it. For each of these parameter values, we generated 5 networks each, and used the average of the metrics obtained as a result, eliminating potential outliers and exceptions that might bias the ultimate results as the algorithm is probabilistic in nature.

Figure 2 show the values of average path length, average clustering coefficient and power-law coefficient(alpha) for all the generated graphs. This is to demonstrate that our graphs are indeed small world and scale free networks. All the graphs have an average path length between 3 and 7. Path lengths of around 7 are observed in networks where we introduce inter-cluster edges with a probability of 0.01 . This results in clearly separated communities with very little inter-cluster edges, which in turn results in increased distances among nodes from different clusters. For the clustering coefficient values, we used two parameter values, 0.5 where triads are formed only for $50 \%$ newly added nodes with $m$ edges giving $\mathrm{CC}$ values in the range of 0.3 and 0.5 , and the parameter value 1.0 where all nodes and edges added to the network belong to at least one triad which raises the $\mathrm{CC}$ around 0.65. Finally for the alpha value, since all our connectivity is based on preferential attachment, all the 32 generated networks have values between 2 and 3 .

In order to show the presence of community structures in the generated networks, we used two well known metrics, Modularity (Q) [39] and Relative Density [40]. We clustered the generated graphs using the method proposed by Newman [41] which generates flat clusters. We calculated the Q and $\mathrm{RD}$ values which are shown in figure 3 . Consistently high values clearly demonstrate the presence of community structures present in the networks. As the inter-cluster edges are increased, both $\mathrm{Q}$ and $\mathrm{RD}$ values decrease implying that the control parameter $P_{c}$ can be used to generate communities with low or high inter-cluster edges which subsequently affects the $\mathrm{Q}$ and $\mathrm{RD}$ values.

Figure $4(a, c)$ are two sample networks (Network 5 and 

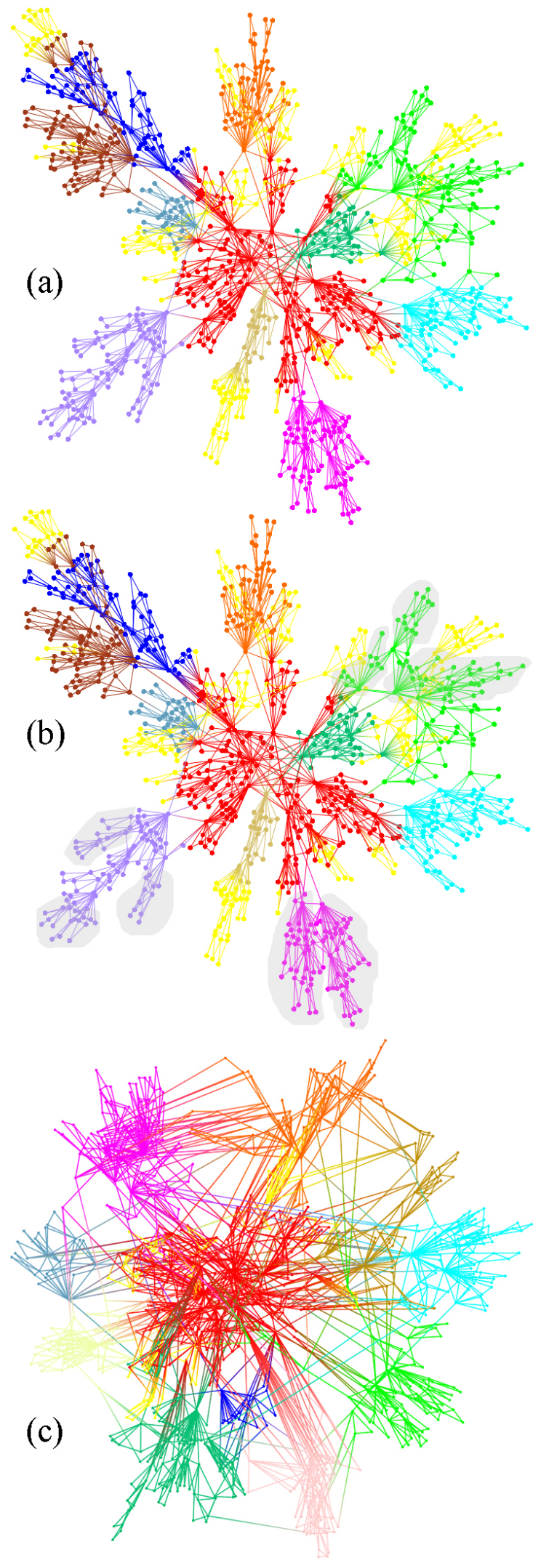

Fig. 4. Figure shows the graphical representation of the networks generated using the proposed model. (a) Network 5 with clearly seperated communities. The top 10 communities in terms of size are uniquely colored and smaller communities are colored in yellow. (b)Network 5 with smaller communities outlined within larger communities. (c) Network 6 with more inter-cluster edges and less seperation between communities.

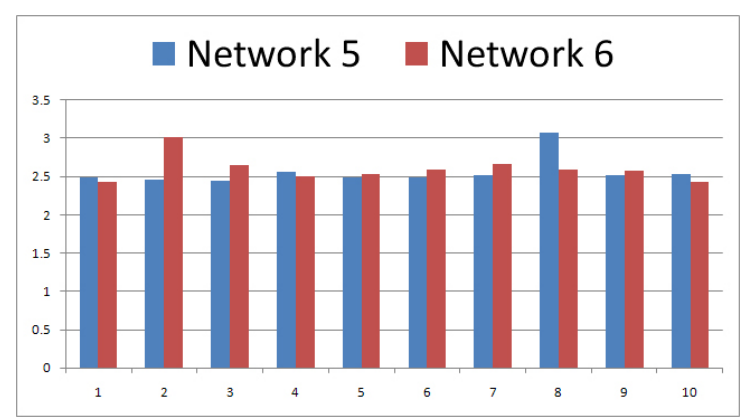

Fig. 5. The graph shows the values of power-law coefficient for the top 10 communities in terms of size for the two networks shown in 4 All the values are consistently between 2 and 3 aprrox. Network 5 has parameters: $n=1000, c=10, P_{t}=1.0, P_{c}=0.01$ and Network 6 with parameters: $n=$ $1000, c=10, P_{t}=1.0, P_{c}=0.1$.

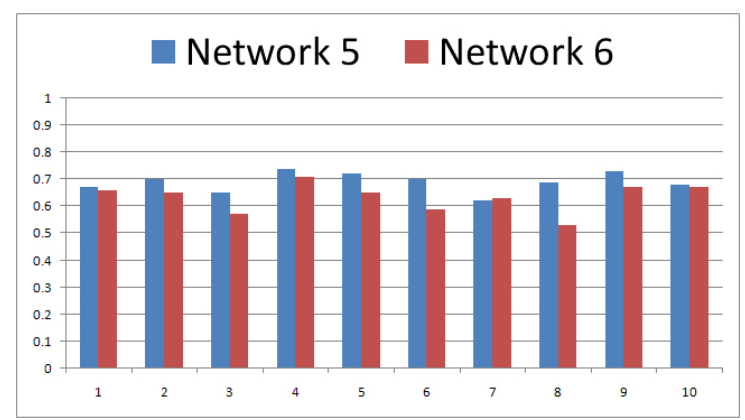

Fig. 6. The graph shows the values of clustering coefficient for the top 10 communities in terms of size for the two networks shown in 4 All the values are consistently above 5 . Network 5 has parameters: $n=1000, c=10, P_{t}=$ $1.0, P_{c}=0.01$ and Network 6 with parameters: $n=1000, c=10, P_{t}=$ $1.0, P_{c}=0.1$.

6 in Table I) generated from the proposed model. Both these networks show community structures with different color encodings for the top 10 communities in terms of size. More communities with smaller sizes are encoded with yellow color.

Figure 4 b) shows Network 5 where larger clusters contain clear separation and can be re-clustered to form hierarchical community structures. Some of these clusters are highlighted and can be visually compared with Figure 4 (a).

To prove our claim that the communities thus produced follow power-law degree distribution with high clustering coefficient, we plotted the power-law coefficient and clustering coefficient of the top ten communities in terms of node-size for the two graphs presented in figure 4. Figure 5 shows the power-law coefficient which lies in the range [2,3] clearly showing that the communities thus produced follow scale free behavior. Similarly figure 6 shows high clustering coefficient values for the biggest communities in networks 5 and 6 reaffirming that formation of triads ensures that communities have a large number of triads.

Finally to show the scalability of the proposed model, we plot the execution times for the generation of different size networks in Table $\Pi$. The proposed model has been implemented using Tulip graph library ${ }^{1}[42]$. The running

\footnotetext{
${ }^{1}$ The source code of the model can be requested from the first author through email.
} 


\begin{tabular}{|l|c|c|}
\hline S.No & Nodes & Time in Seconds \\
\hline \hline 1 & 1000 & below 1 \\
\hline 2 & 10000 & 2 \\
\hline 3 & 100000 & 224 \\
\hline 4 & 1000000 & 21966 \\
\hline
\end{tabular}

TABLE II. THE TABLE SHOWS THE RUNNING TIMES IN SECONDS FOR GENERATING DIFFERENT SIZE NETWORKS USING THE PROPOSED MODEL.

times given in the table are on a standard intel core i5 machine with 4Gb Ram.

In terms of complexity of the proposed algorithm, the most complex task is the calculation of probability for a new node entering the network based on preferential attachment. This task, in the worst case requires $n * n c_{\text {large }}$ steps where $n$ is the desired number of nodes in the network and $n c_{\text {large }}$ is the number of nodes in the largest community. The preferential attachment probability for every node added to the network is calculated in the worst case, with every other node in the largest community. If a network is generated with only a single community, $n c_{\text {large }}$ becomes $n$ and the complexity of the entire algorithm would then become $O\left(n^{2}\right)$. For a network where the community sizes are sparsely distributed, as is the case with many real world networks, this complexity becomes $O\left(n * n c_{\text {large }}\right)$ with $n c_{\text {large }}<<n$.

\section{CONCLUSION}

In this paper, we have introduced a new tunable and growing network generation model which incorporates the well known small world and scale free properties as well as the presence of community structures. The model incorporates three important features of community structures from our society. First, the node degree distribution within a community follows power-law behavior, second, the clustering coefficient within communities is high and finally there is a hierarchical community structure within communities. The model is very flexible and robust and can be used to generate a variety of networks as per requirements. These characteristics can be very useful for generating benchmark and test datasets for empirical studies. Although the model is flexible, but it does not include domain dependent knowledge and cannot be used to generated networks with structural properties other than community structures, small world, scale free and random. This work can clearly be extended to generate networks for particular domains such as biological networks, computer networks. Further more, the current model does not simulate the dynamic changes like removal of previously added nodes or edges, changing previously added edges which are important characteristics for the recently studied social networks. We intend to extend this study towards this direction as well.

\section{REFERENCES}

[1] P. Erdös and A. Rényi, "On random graphs, i," Publicationes Mathematicae (Debrecen), vol. 6, pp. 290-297, 1959

[2] D. J. Watts and S. H. Strogatz, "Collective dynamics of 'small-world' networks," Nature, vol. 393, pp. 440-442, Jun. 1998.

[3] A. L. Barabási and R. Albert, "Emergence of scaling in random networks," Science, vol. 286, no. 5439, pp. 509-512, 1999.

[4] D. J. Watts, Six Degrees: The Science of a Connected Age, 1st ed. W. W. Norton \& Company, February 2003

[5] S. N. Dorogovtsev and J. F. F. Mendes, "Evolution of networks," Advances in Physics, vol. 51, pp. 1079-1187, Jun. 2002.
[6] M. E. J. Newman, "The structure and function of complex networks," SIAM Review, vol. 45, p. 167, 2003.

[7] F. Gilbert, P. Simonetto, F. Zaidi, F. Jourdan, and R. Bourqui, "Communities and hierarchical structures in dynamic social networks: analysis and visualization," Social Network Analysis and Mining, vol. 1, pp. 83-95, 2011.

[8] S. Lim, C. Yu, and C. Das, "Clustered mobility model for scale-free wireless networks," Nov. 2006, pp. 231-238.

[9] A. Wagner and D. Fell, "The small world inside large metabolic networks," Aug. 212000.

[10] C. Ducruet and F. Zaidi, "Maritime constellations: a complex network approach to shipping and ports," Maritime Policy \& Management, vol. 39, no. 2, pp. 151-168, 2012.

[11] K. A. Eriksen, I. Simonsen, S. Maslov, and K. Sneppen, "Modularity and extreme edges of the internet," Physical review letters, vol. 90, no. 14, p. 148701, 2003.

[12] R. Lambiotte and P. Panzarasa, "Communities, knowledge creation, and information diffusion," Journal of Informetrics, vol. 3, no. 3, pp. 180190, Sep. 2009.

[13] N. G. Becker and S. Utev, "The effect of community structure on the immunity coverage required to prevent epidemics," Mathematical biosciences, vol. 147, no. 1, pp. 23-39, 1998.

[14] A. Lancichinetti, S. Fortunato, and F. Radicchi, "Benchmark graphs for testing community detection algorithms," Physical Review E, vol. 78, no. 4, p. 046110, 2008.

[15] A. Lancichinetti and S. Fortunato, "Benchmarks for testing community detection algorithms on directed and weighted graphs with overlapping communities," Physical Review E, vol. 80, no. 1, p. 016118, 2009.

[16] M. Girvan and M. E. J. Newman, "Community structure in social and biological networks," Proc. Natl. Acad. Sci. USA, vol. 99, pp. 82718276, 2002.

[17] P. Holme and B. J. Kim, "Growing scale-free networks with tunable clustering," Physical Review E, vol. 65, p. 026107, 2002.

[18] M. Molloy and B. Reed, "A critical point for random graphs with a given degree sequence," Random Structures and Algorithms, vol. 6, pp. 161-180, 1995.

[19] J.-G. Liu, Y.-Z. Dang, and Z. tuo Wang, "Multistage random growing small-world networks with power-law degree distribution," Chinese Phys. Lett., vol. 23, no. 3, p. 746, Oct. 312005.

[20] P. Fu and K. Liao, "An evolving scale-free network with large clustering coefficient," in ICARCV. IEEE, 2006, pp. 1-4.

[21] K. Klemm and V. M. Eguiluz, "Growing scale-free networks with small world behavior," Physical Review E, vol. 65, p. 057102, 2002.

[22] M. Catanzaro, G. Caldarelli, and L. Pietronero, "Assortative model for social networks," Physical Review E (Statistical, Nonlinear, and Soft Matter Physics), vol. 70, no. 3, pp. 1-4, 2004.

[23] J. Wang and L. Rong, "Evolving small-world networks based on the modified ba model," Computer Science and Information Technology, International Conference on, vol. 0, pp. 143-146, 2008.

[24] Y. Li, X. Qian, and D. Wang, "Extended hk evolving network model," in Control and Decision Conference (CCDC), 2012 24th Chinese. IEEE, 2012, pp. 4095-4097.

[25] M. E. J. Newman, D. J. Watts, and S. H. Strogatz, "Random graph models of social networks," Proceedings of the National Academy of Sciences of the United States of America, vol. 99, no. Suppl 1, pp. 2566-2572, February 2002.

[26] J.-L. Guillaume and M. Latapy, "Bipartite graphs as models of complex networks," in Workshop on Combinatorial and Algorithmic Aspects of Networking (CAAN), LNCS, vol. 1, 2004.

[27] S. Bu, B.-H. Wang, and T. Zhou, "Gaining scale-free and high clustering complex networks," Physica A: Statistical Mechanics and its Applications, vol. 374, pp. 864-868, 2007.

[28] M. E. J. Newman, "Models of the small world," Journal of Statistical Physics, vol. 101, pp. 819-841, 2000, 10.1023/A:1026485807148.

[29] J. Badham and R. Stocker, "A spatial approach to network generation for three properties: degree distribution, clustering coefficient and degree assortativity," Journal of Artificial Societies and Social Simulation, vol. 13, no. 1, p. 11, 2010. 
[30] F. Zaidi, "Small world networks and clustered small world networks with random connectivity," Social Network Analysis and Mining, vol. Volume 3, no. 1, pp. 51-63, 2013.

[31] C. Li and G. Chen, "Modelling of weighted evolving networks with community structures," Physica A: Statistical Mechanics and its Applications, vol. 370, no. 2, pp. 869-876, 2006.

[32] Z. Xie, X. Li, and X. Wang, "A new community-based evolving network model," Physica A: Statistical Mechanics and its Applications, vol. 384, no. 2, pp. 725-732, 2007.

[33] X. Zhou, L. Xiang, and W. Xiao-Fan, "Weighted evolving networks with self-organized communities," Communications in Theoretical Physics, vol. 50, no. 1 , p. 261, 2008.

[34] J. Kumpula, J.-P. Onnela, J. Saramäki, J. Kertesz, and K. Kaski, "Model of community emergence in weighted social networks," Computer Physics Communications, vol. 180, no. 4, pp. 517-522, 2009.

[35] X.-J. Xu, X. Zhang, and J. Mendes, "Growing community networks with local events," Physica A: Statistical Mechanics and its Applications, vol. 388, no. 7, pp. 1273-1278, 2009.

[36] F.-X. Ren, H.-W. Shen, and X.-Q. Cheng, "Modeling the clustering in citation networks," Physica A: Statistical Mechanics and its Applications, 2012.

[37] P. Moriano and J. Finke, "On the formation of structure in growing networks," arXiv preprint arXiv:1301.4192, 2013

[38] A. Sallaberry, F. Zaidi, and G. Melançon, "Model for generating artificial social networks having community structures with small-world and scale-free properties," Social Network Analysis and Mining, pp. 113, 2013.

[39] M. E. J. Newman and M. Girvan, "Finding and evaluating community structure in networks." Phys Rev E Stat Nonlin Soft Matter Phys, vol. 69, no. 2 Pt 2, p. 026113, February 2004. [Online]. Available: http://view.ncbi.nlm.nih.gov/pubmed/14995526

[40] S. E. Schaeffer, "Graph clustering," Computer Science Review, vol. 1, no. 1, pp. 27-64, August 2007. [Online]. Available: http://dx.doi.org/10.1016/j.cosrev.2007.05.001

[41] M. E. J. Newman, "Finding community structure in networks using the eigenvectors of matrices," Physical Review E (Statistical, Nonlinear, and Soft Matter Physics), vol. 74, no. 3, 2006.

[42] D. Auber, "Tulip - a huge graph visualization framework," in Graph Drawing Software, ser. Mathematics and Visualization Series, P. Mutzel and M. Jünger, Eds. Springer Verlag, 2003. 